isolé de surveiller la partie visible du ciel, qu'à ceux qui étaient situés plus haut, à Dailly, où l'horizon est sensiblement plus étendu.

On ne pouvait pas davantage demander, cette nuit là, aux observateurs, de déterminer les radiants des météores. Ils ont seulement noté que les étoiles filantes venaient du $\mathrm{N}$, du NE, et aussi du NW. Mais l'énorme augmentation de leur nombre sur celui qui avait été constaté la veille, prouve que c'étaient, en grande majorité, des Léonides. L'intensité maximum du passage des étoiles filantes s'est manifestée le 15 au matin, entre $5^{\text {h }}$ et $5^{1} /^{h}$, car on a noté, durant ce quart d'heure, 2 I météores à Dailly et 29 à Savatan.

D'aprés les indications des observateurs, les météores étaient brillants et même très brillants. Ils ne laissaient généralement pas de traînées lumineuses dans l'air. La

Observatoire de Genève, 1899 Janvier. couleur des étoiles filantes a été notée comme plutôt jaunâtre et même rougeâtre.

En ce qui concerne les Biélides, les renseignements qui nous viennent de St. Maurice ne nous apprennent pas grand chose. A Savatan, le brouillard a régné, comme dans la vallée du 22 au 24 Novembre. Il en a été de même à Dailly durant la première nuit. Mais, dans la nuit du 23 au 24, il y a eu quelques éclaircies dans le brouillard, et les observateurs, qui s'étaient postés au sommet de la montagne, à $1500 \mathrm{~m}$ de hauteur, ont pu noter 10 météores de minuit à $11 / 2^{\mathrm{h}}$ et 5 de $2^{\mathrm{h}} 20^{\mathrm{m}}$ à $3^{\mathrm{h}} 45^{\mathrm{m}}$. Il est impossible de dire si c'étaient des Biélides. Les observateurs ont seulement remarqué que ces étoiles filantes étaient beaucoup moins brillantes que celles des nuits du I 3 au I $_{5}$ Novembre.

A Genève, le temps a été constamment brumeux ou couvert aux deux époques des passages des Léonides et des Biélides.

R. Gautier.

\title{
New Nebulae and Nebulous Stars.
}

(Harvard College Observatory Circular No. $3^{8}$ ).

Much care and skill are required to obtain the best results with the Bruce photographic telescope. Dr. De Lisle Stewart, who has had charge of this instrument for the last year, has succeeded in obtaining nearly circular images even when the exposures extended over several hours. He has recently found an interesting group of nebulae, hitherto unknown, within the limits of right ascension, $3^{\mathrm{h}} 10^{\mathrm{m}}$ to $3^{\mathrm{h}} 50^{\mathrm{m}}$ ( 1900 ), and declination, $-49^{\circ} 50^{\prime}$ to $-53^{\circ} 40^{\prime}$ (1900).

A comparison of two plates, A 3339 , and A 3346 , taken

\begin{tabular}{|c|c|c|c|}
\hline No. & $\alpha 1900$ & $\delta \mathrm{rg} 90$ & Description \\
\hline $\mathbf{I}$ & $3^{\mathrm{h}} 10^{\mathrm{m}} .0$ & $-50^{\circ} 58^{\prime}$ & 2 faint elong. neb. \\
\hline 2 & $13 \cdot 7$ & $5 \mathrm{I}$ & Elong. $n$ to $\mathrm{s}$, small. \\
\hline 3 & 16.0 & $49 \quad 57$ & Spiral? \\
\hline 4 & 16.7 & 513 & Elong. $\mathrm{n}$ to $\mathrm{s}$. \\
\hline 5 & 17.2 & 5233 & Double, elong. sp to $\mathrm{nf}$. \\
\hline 6 & 19.4 & 5333 & Elong. $\mathrm{n}$ to $\mathrm{s}$. \\
\hline 7 & 21.4 & 534 & Stellar. \\
\hline 8 & 21.7 & $5^{x} 5$ & Stellar. \\
\hline 9 & 21.7 & 513 & Stellar. \\
\hline Io & 21.8 & $50 \quad 55$ & Stellar. \\
\hline I I & 22.1 & $5^{2}$ & Elong., stellar. \\
\hline 12 & 22.3 & 5137 & Elong. $\mathrm{np}$ to $\mathrm{sf}$, stellar. \\
\hline 13 & $22 \cdot 3$ & $5^{2} 3$ & Elong., stellar. \\
\hline I 4 & $22 \cdot 3$ & $5^{2}$ & Very faint. \\
\hline 15 & 22.5 & 5137 & Elong. np to sf. \\
\hline I 6 & 22.9 & $5 \times 41$ & Elong. $\mathrm{n}$ to $\mathrm{s}$ \\
\hline 17 & 22.9 & 538 & Ellip. elong. sp to $\mathrm{nf}$. \\
\hline 18 & 23.1 & $50 \quad 22$ & Stellar, elong. spiral? \\
\hline 19 & 23.5 & 5 I 40 & Stellar, elong. $n p$ to $s f$. \\
\hline 20 & 24.4 & 5322 & Perhaps double star. \\
\hline 2 I & 24.8 & 5125 & Elong. $p$ to $f$ \\
\hline 22 & 24.8 & 5229 & Neb. star. \\
\hline 23 & 325.2 & $-53 \quad$ I & Stellar, elong. $\mathbf{n}$ to $\mathbf{s}$. \\
\hline
\end{tabular}

on October I 4 and October 20, I 898 , respectively, with exposures of four hours each, shows the presence of the objects given in the following table. The current number assigned to each object is given in the first column, the approximate right ascension and declination for 1900 , in the second and third, and a brief description of the object in the fourth column. The letters $n, s, p$, and $f$, in the fourth column are used to indicate, north, south, preceding, and following, respectively.

\begin{tabular}{|c|c|c|c|}
\hline No. & $\alpha 1900$ & $\delta \times 900$ & Description \\
\hline 24 & $3^{\mathrm{h}} 26^{\mathrm{m}} \cdot 5$ & $-5^{2^{\circ}} 59^{\prime}$ & Stellar. \\
\hline 25 & 26.6 & $5^{2} 5^{8}$ & Stellar. \\
\hline 26 & 27.6 & 5040 & Stellar. \\
\hline 27 & 27.7 & $50 \quad 39$ & Spiral ? \\
\hline 28 & 27.7 & $50 \quad 37$ & Star prec. \\
\hline 29 & $28 . \mathrm{I}$ & $5 \circ 4^{6}$ & Elong. $n p$ to $s f$. \\
\hline 30 & 28.3 & 5329 & Elong. sp to $\mathrm{nf}$. \\
\hline 3 I & 28.6 & $5^{2}$ I 5 & Fine small spiral. \\
\hline $3^{2}$ & 29.4 & 5247 & Elong. sp to $\mathrm{nf}$. \\
\hline 33 & 29.9 & 5147 & Stellar. \\
\hline 34 & 30.2 & $50 \quad 45$ & Elong. $\mathrm{np}$ to $\mathrm{sf}$. \\
\hline 35 & 30.9 & 5330 & Elong. $p$ to $f$. \\
\hline $3^{6}$ & $3 \mathrm{r.2}$ & 5139 & Stellar. \\
\hline 37 & 31.8 & 5058 & Stellar. \\
\hline $3^{8}$ & 33.2 & $52 \quad 58$ & Elong. $p$ to $f$. \\
\hline 39 & 33.6 & 52 I 8 & Elong. \\
\hline 40 & 33.6 & 5219 & Elong. \\
\hline 41 & 33.7 & $49 \quad 54$ & Elong. $n p$ to $s f$. \\
\hline 42 & 34.1 & 5029 & Elong. $\mathrm{n}$ to $\mathrm{s}$. \\
\hline 43 & 39.1 & $51 \quad 17$ & Stellar. \\
\hline 44 & $4 I .9$ & $5 \mathrm{I} 5 \mathrm{I}$ & Stellar, elong. $\mathrm{sp}$ to $\mathrm{nf}$ \\
\hline 45 & $42 \cdot 3$ & 5119 & Stellar. \\
\hline 46 & 343.0 & $-5^{1} 5^{8}$ & Elong. $\mathrm{n}$ to $\mathrm{s}$. \\
\hline
\end{tabular}


Only two nebulae are given, in this region, in Dreyer's New General Catalogue. NGC. 13 I is identical with No. 5, and NGC. 1356 is identical with No. 27.

It will be noticed that four of these nebulae appear to be spiral. No. 3 is described as $\gg$ bright elongated centre, faint nebulous wisps in ellipse or spiral. \& No. I 8 stellar

Harvard College Observatory, 1899 Jan. 3 I. nucleus with elliptical nebulosity sp.\& No. 27 Faint nebulous star surrounded by nebulosity. One wisp has spiral tendency. Two nebulous stars sp. and sf., very close to main nebulosity." No. $3^{I}$ "Very fine small spiral nebula with two branches. «

A bright meteor trail appears on Plate A 3346.

Edward C. Pickering.

\section{Ueber die Bewegung des Sterns 9 Ursae majoris in der Gesichtslinie.}

Ich erlaube mir, die Aufmerksamkeit der Spectroscopisten auf den Stern $\vartheta$ Ursae majoris $\left(\alpha=9^{\mathrm{h}} 26^{\mathrm{m}}\right.$, $\delta=+52^{\circ} 8^{\prime}, 3^{\mathrm{m}}$ 2) zu lenken. Diesen Stern habe ich im Jahre I 894 zweimal, am 24. und 27. März, spectrographisch untersucht (mittelst des 30-Zöllers und eines Spectrographen mit 2 Prismen). Diese Aufnahmen zeigten einen Unterschied in den Geschwindigkeiten im V. R. von $2.88 \mathrm{~g} . \mathrm{M}$. Der Stern gehört zu dem II. Typus, deswegen ist der gefundene Unterschied schwer einem Fehler zuzuschreiben; da aber die Platte vom 24. März etwas schwach ist, so wagte ich damals nicht den Schluss zu ziehen, dass wir es mit einem Stern mit veränderlicher Geschwindigkeit zu thun haben.

Im Jahre 1896 erhielt ich 4 Spectrogramme des Sterns, von denen 3 auf keine merkbare Veränderung der Geschwindigkeit im V. R. deuten; die vierte aber giebt entschieden eine stark abweichende Geschwindigkeit. Zufälligerweise war das letzte Spectrogramm seit 1896 bis jetzt nicht ausgemessen worden.

Der Stern scheint hiernach eine periodische Geschwindig. keit zu haben mit einer Periode von etwa 5 bis 7 'Tagen. Im folgenden sind die Resultate der Messungen gegeben:

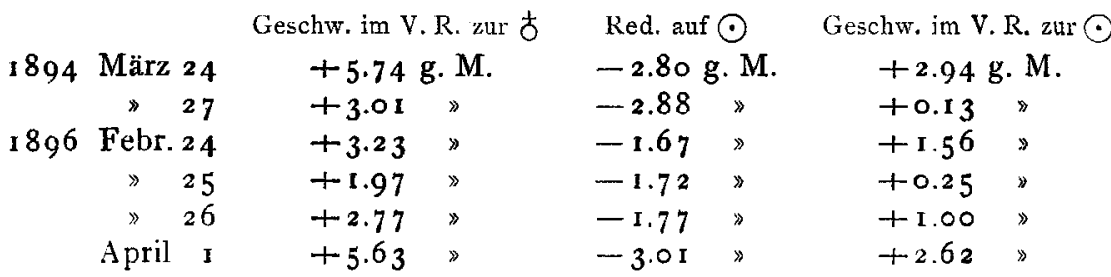

Die Platten vom Jahre 1894 wurden unabhängig von mir und H. Orbinsky gemessen; alle andern von mir allein. Pulkowo, 1899 Febr. 20.

A. Belopolsky.

\section{Notiz zu U Draconis und X Geminorum.}

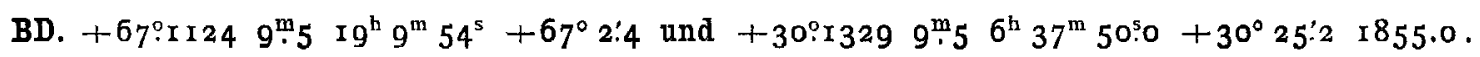

Für die Bestimmung der Periode des neueren Andersonschen Veränderlichen U Draconis liefern die BD.-Zonen einen werthvollen Beitrag. Der Stern ist hier beobachtet:

Rev. Zone 474 186I April I8 Krueger $9^{\mathrm{m}} \cdot 5$ I $9^{\mathrm{h}} 9^{\mathrm{m}} 53^{\mathrm{s}} \cdot 7+67^{\circ} 2 ! 5$

Rev. Zone 4761861 April 19 Krueger $9^{\mathrm{m}} \cdot 519^{\mathrm{h}} 9^{\mathrm{m}} 53^{\mathrm{s}} \cdot 7+67^{\circ} 2: 3$.

Der Stern ist hiernach im April 1861 in der Nähe des Maximums gewesen. Ausserdem sind die folgenden Zonen über den Ort gegangen:

Z. I 425 Schönfeld 1857 Nov. I5. Sehr klare, doch etwas feuchte Luft. Der Stern fehlt.

Z. 1406 Krueger I 857 Nov. Io. Luft sehr klar. Der Stern fehlt. Es war reichlich Zeit zur Beobachtung.

Z. I $_{383}$ Krueger 1857 Oct. 17 . Luft ganz gut, Ocular aber oft angelaufen, wodurch das deutliche Sehen erschwert wurde. Der Stern fehlt. Die Gegend ist aber so sternarm, dass das Fehlen des Sterns doch auffällig ist, obwohl er an der Nordgrenze der Zone passiren musste. Bonn, 1899 Jan. 9.
Bruchst. 1857 Nov. 6. Luft dunstig. Der Stern fehlt.

Aus dem übereinstimmenden Fehlen des Sterns in diesen vier, zeitlich nahe bei einander liegenden Zonen folgt mit überwiegender Wahrscheinlichkeit, dass der Stern 1857 Oct.-Nov. vom Maximum der Helligkeit beträchtlich entfernt war.

$\mathbf{X}$ Geminorum ist hier beobachtet :

Z. 868 Kr. $185^{6}$ März 25. $9^{\mathrm{m}} \cdot 56^{\mathrm{h}} 37^{\mathrm{m}} 4^{8} \cdot 5+30^{\circ} 26^{\mathrm{s}} \mathrm{r}$. Luft meist gut, zuweilen vielleicht etwas dunstig.

Rev. Z. 175 Sch. 1858 Jan. $21.9^{\mathrm{m}} \cdot 76^{\mathrm{h}} 37^{\mathrm{m}} 50^{\mathrm{s}} \cdot 3+30^{\circ} 25^{\prime} \cdot 2$. Meist sehr schöne Luft; Mondschein noch ziemlich schwach.

Ausserdem gingen folgende Zonen über den Ört:

Z. 1163 Sch. 1857 Febr. 16. Der Stern fehlt. Wechselnde, selten ganz klare Luft.

Z. I I 42 Sch. I 857 Jan. 20 . Der Stern fehlt. Anfangs $\left[5^{\mathrm{h}} 3 \mathrm{I}^{\mathrm{m}} \mathrm{2}\right]$ klare, später feuchte Luft.

Fr. Deichmüller. 